\title{
CC Ost kooperiert mit Automotive Cluster Ostdeutschland e.V.
}

Die Abteilung CC Ost des Augsburger Carbon Composites e.V. (CCeV) hat eine Kooperationsvereinbarung mit dem Automotive Cluster Ostdeutschland e.V. (ACOD) geschlossen. Beide Organisationen setzen sich für die Stärkung von Forschung und Industrie auf dem Gebiet der Hochleistungs-Faserverbundstrukturen für Automobilanwendungen ein.

Der Anfang 2004 gegründete und 34 Mitgliedsunternehmen umfassende ACOD ist eine länderübergreifende Initiative zur nachhaltigen Entwicklung der Automobilindustrie in Ostdeutschland. In dem Cluster sind Automobilhersteller, Zulieferer und Dienstleister, Forschungsinstitute, Verbände und andere Institutionen der fünf neuen Bundesländer organisiert. Die Regionalabteilung CC Ost des CCeV hat ein ähnliches Einzugsgebiet und bündelt die Faserverbundkompetenzen von Unternehmen und Forschungseinrichtungen. Sowohl ACOD als auch CC Ost setzen sich für eine Stärkung von For- schung und Industrie auf dem Gebiet der Hochleistungs-Faserverbundwerkstoffe für Automobilanwendungen ein.

Im Rahmen ihrer Kooperation wollen die beiden Organisationen Strategien erarbeiten und umsetzen, mit denen die Weiterentwicklung von Hochleistungs-Faserverbundwerkstoffen und deren Einsatz in der Automobilindustrie vorangetrieben werden soll. Geplant sind auch gemeinsame Aktivitäten, um die Aufmerksamkeit einer breiteren Öffentlichkeit zu intensivieren. Darüber hinaus wird eine gemeinsame Interessenvertretung in nationalen und internationalen Gremien ins Auge gefasst.

Um die gemeinsamen Ziele zu erreichen, werden sich Vertreter von ACOD und CC Ost regelmäßig treffen und abstimmen. Für die Mitglieder beider Organisationen ist die Zusammenarbeit in Kompetenzclustern des ACOD beziehungsweise Arbeitsgruppen des CC Ost vorgesehen. „Durch die enge Vernetzung des leistungsstarken Automoti-
ve-Sektors mit den ausgewiesenen Faserverbundkompetenzen im ostdeutschen Raum haben wir die Chance, unsere gemeinsamen Ziele unter Nutzung vorhandener und zukünftiger Synergien intensiv zu verfolgen", unterstreicht Dr. Thomas Heber, Geschäftsführer des CC Ost.

Der CCeV wurde 2007 gegründet und zählte Ende September 2012170 Mitglieder, darunter 34 Forschungseinrichtungen, 41 Großunternehmen, 87 kleine und mittlere Unternehmen sowie sieben unterstützende Organisationen. Die Abteilung CC Ost des CCeV dient zur Stärkung und Bündelung der Faserverbund-Kompetenzen im ostdeutschen Raum sowie als regionale Interessenvertretung. Der Gründungsvorstand besteht aus dem Vorsitzenden Prof. Werner Hufenbach, Direktor des Instituts für Leichtbau und Kunststofftechnik, und Prof. Jens Ridzewski, Abteilungsleiter Kunststoffe der IMA Materialforschung und Anwendungstechnik GmbH.

\section{REGIONALABTEILUNG SÜDWEST \\ CARBON COMPOSITES GRÜNDET NEUE VERTRETUNG}

Am 10. Dezember 2012 wurde die neue Regionalabteilung Südwest des Carbon Composites (CCeV) ins Leben gerufen. Die Gründungsversammlung fand im Institut für Verbundwerkstoffe (IVW) in Kaiserslautern statt.

Die neue Regionalabteilung strebt einen Industrie- und Forschungsverbund mit etwa 30 Mitgliedern in den kommenden drei Jahren an.

Uwe Hüser, Staatssekretär im Ministerium für Wirtschaft, Klimaschutz, Energie und Landesplanung, betonte die zusätzlichen Chancen für zielgerichtete regionale Kooperationen zwischen Wissenschaft und Wirtschaft, die der CCeV zum Ziel hat. Vor allem kleine und mittelständische Unternehmen könnten von der neuen regionalen Geschäftsstelle profitieren.

Das IVW in Kaiserslautern ist eine 1990 gegründete Forschungseinrichtung des Landes Rheinland-Pfalz. Mehr als 100 Wissenschaftler und Techniker arbeiten an der Entwicklung neuer Composite-Anwendungen. Jährlich werden etwa 200 Projekte mit über 400 internationalen Partnern entlang der Wertschöpfungskette bearbeitet. Im Vordergrund stehen energieeffiziente und ressourcensparende Composite-Anwendungen für den Au- tomobilbau, die Luft- und Raumfahrt, den Maschinenbau, Windkraftanlagen, Sportund Freizeitgeräte, Medizintechnik und viele weitere Branchen. Dafür werden neue, multifunktionale Werkstoffe untersucht und mit modernen Analyseverfahren auf ihre Eignung geprüft. Für die anwendungsoptimierte Konstruktion werden neue Auslegungsmethoden und Bauweisen entwickelt. Einen weiteren Schwerpunkt bildet die Verarbeitungstechnik. Hier werden im Maßstab 1:1 neue Verfahren konzipiert und erprobt, die eine effiziente Produktion von Verbundwerkstoffbauteilen in großen Stückzahlen erlauben. 